Even if centrifugation does not cause aggregation, completely unaggregated preparations could be expected only from plants that have been recently infected, for aggregation occurs naturally in the sap of plants that have been long infected ${ }^{4}$.

$W_{\theta}$ have emphasized the need for caution in interpreting centrifugal data on systems which can, in some circumstances, aggregate. It may be possible to isolate unaggregated tobacco mosaic virus by short periods of centrifuging in strong gravitational fields at a low temperature. But it is significant that four sedimentations cause an increase in anisotropy of flow detectable by the method of Loring, Lauffer and Stanley. It therefore seems improbable that one sedimentation should have no effect. Also, until more adequate data on the activity, chemical composition and physical properties of virus preparations made solely by high-speed centrifugation have been published, it will not be possible to assess their purity.

\footnotetext{
${ }^{\mathrm{t}}$ Bawden, F. C., and Pirie, N. W., Proc. Roy. Soc., B, 123, 309 (1937). ${ }^{2}$ Bawden, F. C., and Pirie, N. W., Reunion Inter. de Physique, Chimie et Biologie (Paris, 1937), p. 382

'Lauffer, M. A., and Stanley, W. M., J. Biol. Chem., 123, 507 (1938).
}

'Wyckoff, R. W. G., J. Biol. Chem., 121, 219 (1937).
DR. Kenneth M. Smith, F.R.S., Potato Virus Research Station, Cambridge, AND W. D. MacClement, Molteno Institute, Cambridge.

DR. Stanley and his colleagues draw the conclusion that tobacco mosaic virus is not aggregating after centrifugation since it passes a membrane of $190 \mathrm{~m} \mu$ average pore diameter. In our ultrafiltration experiments, we have found that this virus, after precipitation at $p \mathrm{H} 3 \cdot 4$, will pass through a membrane of smaller pore size than the foregoing and has an end-point under optimum conditions of 150 $175 \mathrm{~m} \mu$. This indicates an aggregation of some kind, since the accepted ultrafiltration end-point of tobacco mosaic virus in crude clarified sap is about $50 \mathrm{~m} \mu$. In our opinion, therefore, it is not justifiable to draw the conclusion that the virus is not aggregated merely because it passes a membrane of $190 \mathrm{~m} \mu$ average pore diameter. Tobacco mosaic virus, however, which has been subjected to the full process of purification and will therefore take up the liquid crystalline state, has a filtration end-point greater than $450 \mathrm{~m} \mu$; it is clear, therefore, that intermediate stages of aggregation are possible.

\title{
Origin of the Solar System
}

$\mathrm{T}$ HE presidential address to the British Astronomical Association was delivered by Rev. Dr. M. Davidson, on October 26, at Zion College, and dealt with the theories advanced to explain the system of planets and satellites of the solar system.

After brief reference to certain electric theories, in particular to that of H. P. Berlage, which appeared in Prof. Roy. Acad. Amsterdam in 1930-34, other theories which have held the field for a time were discussed. The main objection to the old and rather attractive Laplacian hypothesis is the enormous discrepancy between the angular momentum that the rotating nebula must have possessed and that which the solar system possesses to-day. Further, the theory suggested that the planets were thrown off from the original sun by internal forces and were not removed by some outside agency; but, as the major planets have about 98 per cent of the angular momentum of the solar system and only about 1/700 of the total mass, this suggests the action of an internal body.

The planetismal hypothesis of Chamberlin and Moulton did not receive so much attention in Great Britain as might have been expected. The manner in which the planets were formed, according to this hypothesis, differs essentially from that which the more recent tidal theory assumes. In the former case, they are assumed to have been built up slowly by accretions drawn into the original nuclei; in the latter case the once liquid planets received very little later accretions. The difference may not be of much interest to an astronomer but to the geologist it is of the utmost importance.

The planetismal hypothesis is the parent of the more recent tidal theories, and naturally many of the objections to the latter will also apply to the former. Dealing with Sir James Jeans's theory, which first assumed a very extensive sun but later adopted the 'Roche model'--a body with a strong central con.
densation-Dr. Davidson referred to a number of objections to the theory. The greatest of these, he believes, is that which arises from a consideration of the distribution of angular momentum in the planets, per unit mass, as pointed out by Prof. H. N. Russell. The visiting star which was supposed to have produced the eruption from our sun must have made a very close approach, and, even under the most favourable conditions, it could not have produced ten per cent of the angular momentum per unit mass that the planets possess at present. This seems to be sufficient, without any other objections, to condemn the theory.

The recent theory of Dr. R. A. Lyttleton was then dealt with. This starts with the assumption that the sun was once a binary and that its companion was ejected by a visiting star, the filament formed by the usual disruption, which is supposed to occur in these cases, finally producing the planets and satellites, the latter by close approaches of planets. However, as Dr. H. Jeffreys has shown, the satellites could not have been formed in the manner assumed, and $\mathrm{Dr}$. Lyttleton was forced to abandon his original explanation of these bodies, and to assume rotational instability of enormous primitive planets. Jupiter and Saturn, according to this theory, were once a single extensive planet, and from the filament resulting from the disruption their satellites were formed, and even other independent planets. His explanation of the origin of the primitive planets was severely criticized by Dr. W. J. Luyten and Dr. E. L. Hill. In particular, they had shown that the visiting star, which must have removed the filament from the companion at least 20 astronomical units from the sun (half the distance of Pluto), required a velocity of about $100 \mathrm{~km}$. a second, and at such a distance from the sun the matter would escape. There are other objections, and Dr. Lyttleton, recognizing the validity of these, has modified the 